\title{
LAS ORGANIZACIONES EDUCATIVAS COMO SISTEMAS DE COMUNICACIÓN. UN ENFOQUE MICROPOLITICO
}

\section{Eduardo Terrén $(*)$}

SÍNTESIS: Se ha dicho en repetidas ocasiones que las ciencias sociales son ciencias de lo impreciso. El estudio de la vida de las organizaciones educativas no escapa a esta afirmación, sobre todo cuando el análisis se sitúa más en el terreno de la organización como proceso o acción (organizing), que en el de la organización como estructura o como efecto (organization). La imagen de la organización desarrollada sobre todo desde los enfoques etnográficos o postmodernos y los basados en la asunción del conflicto como algo inherente a la vida de las organizaciones, atiende principalmente a este nivel de producción cultural de la organización en el que se practica el gobierno de los centros educativos. EI presente trabajo aspira a mostrar que la micropolitica es el enfoque a este nivel de análisis. En primer lugar, porque permite mostrar cómo la unidad relevante en el estudio de esa práctica la forman no las estructuras organizativas ni los individuos, sino las redes de interacción que se constituyen entre ambos. En segundo lugar, porque a través del estudio de tales redes adquieren relevancia los procesos de interacción fundamentales que inciden en el gobierno de un centro: el intercambio de información y la creación de sentimientos de confianza. Son estos procesos los que constituyen la base sobre la que se desarrolla el capital social interno de la organización, de los que depende en buena medida su capacidad de adaptación al cambio y de llevar a cabo un proyecto común.

(*) Universidad de La Coruña 


\section{EL ENFOQUE MICROPOLÍTICO DE LA ORGANIZACIÓN EDUCATIVA}

Entre los cambios que conlleva la transformación tecnológica y económica de nuestro tiempo destacan el nuevo carácter y el valor de la información y el conocimiento relevantes. Estamos pasando de un complejo de poder/saber en el que era dominante un modelo de procesamiento vertical de la información basado en la autoridad, a otro en el que el modelo dominante de procesamiento de la información privilegia la rapidez y se desarrolla en red. Este trabajo ha partido de la base de que el estudio del gobierno de las organizaciones educativas debe ser sensible a dicho nuevo valor del conocimiento y de la información, para adquirir una adecuada visión de su verdadero funcionamiento y de su capacidad de adaptación al cambio. Ese es el sentido que tiene nuestro objetivo de explorar el potencial analítico de un marco teórico inspirado en la idea de que los centros educativos pueden estudiarse como sistemas de comunicación, y que, al hacerlo así, lo que estaríamos analizando sería el conjunto de intercambios informativos que fluyen a través de las redes que se originan en su seno. Tal es el escenario de la micropolítica de la organización ${ }^{1}$.

${ }^{1}$ Las reflexiones expuestas aquí se basan en el trabajo realizado en el marco del proyecto de investigación «Configuraciones organizativas y modelos profesionales: análisis de las relaciones entre la profesión, la organización yel entorno escolares», dirigido por el profesor Fernández Enguita (BSO2002-02284, PNICDYT 2002-2003, DGCYT). La evidencia empírica que soporta buena parte de lo aquí propuesto ha sido analizada en Terrén, E. yEsteban, F.: «La gestión del conocimiento yla comunicación en las organizaciones educativas», comunicación presentada en el VIII Congreso Nacional de Sociología, Alicante, septiembre de 2004. 
El gobierno de una organización es el meollo de su política, y toda política es gestión de un determinado conjunto de individuos y de recursos. Los recursos son relevantes en tanto que son conocidos; Io mismo ocurre respecto a los individuos, con la particularidad de que éstos se conocen y se comunican entre sí, formando redes que se traducen en diferentes lógicas de acción. Más que los individuos, son estas redes las que constituyen las unidades significativas del gobierno de las organizaciones, y son los procesos de comunicación implicados en las interacciones registradas dentro de estas redes y de unas redes con otras las que confieren un valor real a los recursos de que dispone la organización ${ }^{2}$. El carácter universal de tal afirmación se hace más elocuente en el caso de las organizaciones escolares (en el fondo instituciones administradoras de conocimiento), y, dentro de ellas, en el de las que pretenden dotarse de un proyecto, asentarse en una cultura colaborativa y desarrollarse como comunidades, pues son éstas las que más precisan de una traslación fluida de informaciones entre sus redes y de un mayor capital relacional.

Analizar el gobierno de una organización pasa por reconocer la gestión del conocimiento y de la comunicación que se producen en su seno como claves de su política. La política de la escuela tiene dos dimensiones: una macro y otra micro. La dimensión macropolítica es la que se refiere a la política educativa en el nivel nacional o regional. Su escenario fundamental es el Estado o la correspondiente entidad política subestatal; su forma de acción es la legislativa, y el tipo de cambio que promueve es la reforma. La dimensión micropolítica, que es la aquí nos interesa, tiene como escenarios privilegiados el centro y el aula, a los que en al gunos casos conviene distinguir como dos subniveles (el micro-meso y el micro-micro, respectivamente), si es que compete distinguir entre los dos tipos de cambio educativo que se promueven en ambas esferas (la mejora y la innovación), en correspondencia con los dos tipos de actuación a los que cada una se corresponde (el gobierno del centro y la práctica de aula). El cuadro 1 sintetiza las diferencias señaladas 3 .

\footnotetext{
${ }^{2}$ La visión de la organización en la que nos proponemos profundizar puede expresarse también en las palabras de Kreps (1990, p 11): «la organización [...] está basada en la cooperación. La cooperación no siempre se logra fácilmente. Hay que persuadir a la gente para que coopere, y la comunicación es una herramienta que ayuda a ello».

${ }^{3}$ Como se observa en el cuadro de referencia, el centro (la unidad ol vidada del cambio hasta hace bien poco) se corresponde más con un nivel meso de cambio, que, no obstante, yaunque a efectos analíticos no deba identificarse, es indisociable del nivel del
} 
CUADRO 1

Dimensiones de la política de la organización educativa

\begin{tabular}{|c|c|c|c}
\hline Nivel & Escenario & Ámbito de acción & Tipo de cambio \\
\hline Macro & Sistema educativo & Legislativa & Reforma \\
\hline Micro-Meso & Centro & $\begin{array}{c}\text { Gobierno de la } \\
\text { organización }\end{array}$ & Mejora \\
\hline Micro-Micro & Aula & Práctica de aula & Innovación \\
\hline
\end{tabular}

En nuestra exposición interesa analizar el nivel micro-meso de la organización educativa, lo que nos sitúa en el ámbito de la micropolítica. Una de las ventajas del análisis a este nivel es que ayuda a eludir la excesiva contraposición entre los enfoques macro y micro del análisis sociológico, centrados respectivamente en el predominio de la estructura o de la acción, pues el enfoque del análisis micropolítico propone una visión del orden de la organización como un orden negociado, en constante proceso, y con una visión de la acción como estructurante y socialmente enraizada en redes y en rutinas (Bacharach y Mundell, 1993; Giddens, 1984).

Esta reflexión se alinea en una tendencia de investigación bien asentada desde hace casi dos décadas. La idea motriz de dicha tendencia consiste en hacer hincapié en que, más allá de las visiones de las escuelas como cajas negras cuyos resultados son interpretados como productos de un proceso simple de input-output sometido al efecto de las variables macro, los propios procesos organizativos de los centros, y, en especial, las variables vinculadas a lo que se conoce como la «cultura de la organización», son factores claves para la consecución de una educación decalidad. La literatura sobre este tema ha puesto de manifiesto dos tesis fundamentales: partiendo del supuesto de que el rendimiento del alumnado está muy condicionado por su motivación y por lo significativo que sea su aprendizaje, se afirma que tal alumnado depende a su vez, y en buena medida, del tipo de cultura organizativa y del clima imperantes en cada escuela; la otra tesis manifiesta que la participación, la implicación y el compromiso con el centro y con sus objetivos por parte tanto del profesorado como de las familias, es parte fundamental de los procesos de mejora de las escuelas. Sólo a efectos analíticos, hablamos de

aula, más estrictamente microsociológico porque la mejora del centro va unida a la mejora (o innovación) de la propia práctica de aula (Fullan, 1990). También Santos Guerra (1997) aboga por esta reducción de ambos niveles. Para un análisis conceptual de los términos referidos a cada nivel de cambio, ver Rodríguez Romero (2003, pp. 80-89). 
colaboración en el primer caso y de participación en el segundo. Ambos se refieren a dos planos distintos aunque interrelacionados de capital social relacional (intra y extraorganizativos), y señalan dos vías de flujo y de alimentación de informaciones (comunicación interna/externa), mostrando una doble extensión de la imagen de la red: hacia adentro (entorno interno) y hacia fuera (entorno externo). Como lo que aquí nos importa es el primer tipo de entorno y su correspondiente desarrollo de capital social o relacional, el sentido de nuestra reflexión puede describirse como una aproximación al estudio del funcionamiento de las redes sociales intraorganizativas, con objeto de mejorar nuestro conocimiento de la micropolítica de colaboración que conlleva el gobierno de los centros educativos dispuestos o enfrentados al cambio.

Conviene subrayar que el análisis de la micropolítica de la organización en función de las interacciones comunicativas que se establecen entre sus redes supone también un particular enfoque de la cultura de la organización, pues, a diferencia de lo que se observa en muchos tratamientos de esta variable, la cultura no se entiende como un conjunto de valores compartidos que en teoría impregnan o deben impregnar el clima de la organización y guiar el desempeño de los individuos que la componen. Seentiende, más bien, que las interacciones comunicativas que alimentan la vida de las diferentes redes constituyen los entornos simbólicos inmediatos en los que los individuos se reconocen y en los que se refugian sus actitudes respecto al trabajo y a la organización, lo que genera corrientes de opinión muchas veces divergentes e incluso contradictorias. Parece plausible afirmar que, desde este punto de vista, la cultura de una organización funciona más como una opinión pública que como una ideología o como una mentalidad común. Tiene sus líderes locales o nod os que focalizan las informaciones, que entretejen en torno a sí opiniones y disposiciones que albergan distintas visiones de su misión, y que filtran informaciones del interior y del exterior con arreglo a sus diferentes historias 0 aspiraciones. Las redes intraorganizativas de interacción no se estructuran sobre roles definidos por una función ni por una asignación de autoridad, sino sobre focos de influencia que canalizan la información, que la elaboran y la estructuran en actitudes y en disposiciones compartidas. Dicho de otra forma, los climas de opinión que emergen de las redes intraorganizativas proporcionan los pseudoentornos en los que se inscriben las lógicas de acción que cada una de ellas impulsa. En la dinámica conflictiva que describe el juego entre estos diversos climas de opinión es en donde se bate la micropolítica. 
Así, y nos parece que en consonancia con tratamientos ya clásicos de la micropolítica como los de Ball (1989) o Blaise (1991), creemos que puede afirmarse que el enfoque micropolítico en el análisis de los centros educativos Ileva al terreno de la cultura de la organización la idea de las organizaciones como sistemas débilmente articulados o acoplados (loosely coupled systems) propuesta hace más de veinte años por Karl Weick. La perspectiva de análisis que aquí se propone (centrada en la relación entre micropolítica, cambio y comunicación), ahonda en la crítica del supuesto de que las organizaciones son entidades monolíticas, burocráticas y férreamente racionales, cuya esencia radica en su estructura. La perspectiva es, más bien, la de que la cultura de la organización es un campo esencialmente conflictivo, en el que compiten o coexisten diferentes interpretaciones y manejos de la situación. La micropolítica de la interacción de redes supone, por tanto, una visión conflictiva de la vida cultural de la organización, que responde a un reconocimiento de su diversidad interna. Esto quiere decir, también, trabajar en la perspectiva de lo que en otro lugar hemos descrito como una «imagen caliente de la organización» (Terrén, 2003).

La micropolítica de la escuela se ocupa tanto de describir esa complejidad interna como del esfuerzo por reducirla y reconducirla, lo que la hace pertinente para el estudio del ejercicio del liderazgo en las organizaciones educativas, entendiendo que tal estudio tiene más que ver con el juego de las influencias frecuentemente desarrolladas a nivel informal, que con el desempeño formal de la autoridad. El estudio de la dimensión específica del gobierno de los centros es muy significativo a la hora de entender que la consideración de la escuela como un espacio micropolítico implica reconocerla como un ámbito de conflicto, en el que se entrecruzan estrategias con diferente capacidad de arrastre en función de los recursos que pueda movilizar. De ello depende su potencial de presión, de apoyo, de negociación y de convicción. Debe apreciarse que, en ese sentido, el conflicto no es tanto una patología que hay que extirpar, sino una situación de hecho que debe saber gestionarse para traducirla en una oportunidad de mejora (Jares, 1997). Cuando hay discrepancias y diferentes niveles de implicación y de compromiso, aumenta la incertidumbre sobre el resultado y sobre los beneficios, y es entonces cuando los procesos de comunicación que fluyen a través de las redes de la organización se convierten en la clave de su micropolítica. 


\section{ORGANIZACIÓN, CONOCIMIENTO Y COMUNICACIÓN}

Organización y comunicación son procesos íntimamente asociados, pues, haciendo uso de la metáfora que McLuhan acuñó para el segundo, ambos pueden considerarse como extensiones del ser humano, que amplían sus posibilidades de adaptación eficiente. No en vano el incremento de la amplitud de dichos procesos y de nuestra dependencia cotidiana de ambos, ha sido repetidamente utilizado como indicador característico de la sociedad de nuestro tiempo, considerada tanto una sociedad de organizaciones como de comunicaciones. Sin embargo, lo importante no está siendo sól o ese incremento sino el de su interconexión o interdependencia, pues la complejidad del mundo de las organizaciones es hoy impensable si no admitimos el mismo grado de complejidad en el mundo de las comunicaciones.

Quizá debido a la interdependencia entre ambos procesos, la gestión del conocimiento y de la comunicación en el seno de las organizaciones se ha convertido en un capítulo fundamental en la literatura sobre el cambio organizativo, dado que, después de todo, la obtención y la transmisión de la información son los recursos fundamentales a la hora de forjar o de reorientar estrategias de adaptación que permitan a las organizaciones responder a las nuevas demandas de su entorno o de su público. Lo que aquí se pretende mostrar es cómo la consideración del papel que cumple la gestión del conocimiento como factor clave del gobierno de las organizaciones escolares es inseparable de la consideración de éstas como espacios comunicativos, y cómo, de acuerdo con lo expuesto en la sección anterior, el análisis de esta gestión puede hacerse con más precisión si se considera la organización como un universo de redes de interacción, y no como una estructura definida de roles o de posiciones preestablecidos.

Este interés se relaciona con el enfoque de la organización de Karl Weick (1979), que habló por primera vez en los años setenta de las organizaciones educativas como sistemas débilmente articulados. A partir de la idea de la reducción de la incertidumbre desarrollada por la teoría de la información de Shanon, y de la importancia concedida a la realimientación en el modelo de la teoría de sistemas, Weick entendía las organizaciones como instrumentos de reducción de la incertidumbre y del carácter equívoco de la información. Su psicología social de la organización, más atenta a los procesos de producción de un orden siempre inacabado ( organizing) que a la estructura resultante, considera los entornos como complejos de información y establece la comunica- 
ción como proceso central de la vida organizativa. Esta visión dinámica, compleja e inacabada de las organizaciones, es quizás la que más ha obligado a llevar al campo de la teoría de la organización el interés por los procesos comunicativos (Kreps, 1995), y es, en cualquier caso, la que proporciona fundamento a una idea central en nuestro enfoque, como es la de que el propio interior de la organización constituye a su vez un entorno (interno) sometido a la misma dialéctica de incertidumbre y de complejidad.

No obstante, puede llegarse a este mismo interés por la vida comunicativa de las organizaciones a través de otro itinerario. Hace tiempo que economistas de la talla de Kenneth Arrow, por ejemplo, subrayaron la importancia del conocimiento y del aprendizaje en el gobierno de las organizaciones. Desde entonces, el interés por la gestión del conocimiento organizacional se ha ido desarrollando a medida que lo han hecho dos tendencias de contextos fundamentales e interrelacionados: la globalización y el desarrollo de las TIC. Así ha ido cuajando la visión de las organizaciones como estructuras dinámicas capaces de aprender y de adaptarse a los cambios a través de unos procesos cada vez más revalorizados, como son el liderazgo efectivo, la cultura colaborativa, el clima abierto y la toma descentralizada de decisiones, mediante la activación de las redes internas de comunicación. La proliferación de entornos más caracterizados por las discontinuidades y por los cambios acelerados que por la continuidad y la estabilidad, ha revalorizado el papel de la gestión del conocimiento en las organizaciones y ha trasladado el centro de interés desde la estructura y la asignación de funciones hacia las personas, y, más específicamente, hacia las redes en las que se producen sus interacciones. La idea básica es que entornos más complejos exigen también estrategias organizativas más complejas, lo que revierte en un incremento de la complejidad interna.

La incorporación de la teoría de la complejidad al análisis de las organizaciones ha subrayado cómo la gestión de la complejidad es ante todo una gestión de la información que fluye a través de las redes. La adecuación entre la complejidad del entorno y la de la organización se obtiene a través de la información, tanto de la procedente del exterior como de la del interior de la organización. La teoría de los sistemas de Luhmann, por ejemplo, permite entender la relación entre organización, complejidad y comunicación ${ }^{4}$. Para Luhmann, las organizaciones, en

\footnotetext{
${ }^{4}$ Luhmann (1983, pp. 294 yss.) propone una visión no teleológica del sistema, que rompe con la idea de que el gobierno de una organización se basa en la planificación
} 
tanto que sistemas, son siempre menos complejas que el entorno, y deben abordar la mayor complejidad de éste a través de representaciones simplificadoras del mismo. Estas representaciones son selecciones de información que, como tales, implican un riesgo en su adecuación. Lo importante de su enfoque para nuestra reflexión es que el riesgo es menor cuando más adaptable, flexible y precisa sea la representación; y eso es lo que hace que los procesos intraorganizativos de selección y de comunicación de las informaciones en sí mismas como de las disposiciones que las acompañan, sean decisivos para el gobierno y para la supervivencia de la organización.

El conocimiento de tales procesos y de sus contenidos es lo que se considera el conocimiento organizacional. No es éste el lugar de extenderse en una revisión de las definiciones y de las tipologías propuestas por la teoría del conocimiento. Para nuestros propósitos basta fundamentar con brevedad una visión del conocimiento adecuada a su papel en la vida y en el gobierno de las organizaciones. La visión que necesitamos se apoya en los presupuestos del pragmatismo, pues relaciona el conocimiento con la capacidad de adaptación al mundo de los individuos y de las organizaciones. De ahí que podamos entender el conocimiento organizacional como el resultado de la selección y del procesamiento de las informaciones que los miembros de la organización tienen de ella y de su entorno. Nuestro conocimiento del entorno comporta una determinada distribución de probabilidades, una cierta expectativa de cómo son las cosas, y, en consecuencia, de cómo es de esperar que sigan siendo. La visión del mundo que ofrece el conocimiento conlleva, por tanto, una determinada orientación del comportamiento basada en una (o varias) reducciones de la complejidad del entorno, pero también en una estructura de relaciones sociales que permiten compartirlo.

Por eso es importante reparar en cómo se relaciona el conocimiento organizacional con el de los integrantes de la organización, y observar que el primero no se deriva del segundo, sino de manera indirecta a través de la interacción conflictiva (o al menos no siempre convergente) de las informaciones procesadas en las diferentes redes de la organización. Como parece ir corroborando nuestra investigación (véase nota 1), la armonización de los procesamientos realizados en cada

y en el control a partir de valores o de fines preestablecidos e invariables. El control se genera para reducir la incertidumbre interna, pero ésta es inevitable a medida que el sistema incrementa su complejidad para responder a la incertidumbre externa. 
una de las redes es una de las tareas claves del liderazgo en las organizaciones, entendidas como sistemas de comunicación. La capacidad de actuación de las organizaciones en términos de su adaptación al entorno no es la suma de las actuaciones de sus integrantes, de la misma manera que la actuación de un equipo deportivo no es el resultado automático de la competencia o de la cualificación de cada uno de sus integrantes. Tampoco una buena escuela es el simple resultado de la suma de las cualificaciones de su profesorado. El conocimiento colectivo no es sólo la agregación de los conocimientos individuales, que deben ser gestionados, esto es, conocidos por los otros y coordinados en función de una estrategia colectiva. Aunque ahora nos centremos en el análisis de dicha gestión, no debe olvidarse que para poder hablar de un auténtico proyecto (es decir, que cuando el compromiso con los objetivos de la organización permite hablar de una comunidad), esa gestión del conocimiento debe ir acompañada de una determinada motivación que podríamos describir como una motivación comunitaria. Es plausible pensar que esta estrategia es más eficaz cuanto más colaboración existe, y que la actitud cooperativa se estimula porque los beneficios que se extraen de ella son percibidos como atractivos. Persuadir de esta atracción es una de las piezas fundamentales del engranaje micropolítico de la colaboración, y en especial del liderazgo, sobre todo del liderazgo de tipo democrático.

Dicha relación es la que en la literatura sobre la gestión de la información en las organizaciones empresariales suele denominarse management estratégico, una de cuyas claves radica en la creación de un conocimiento organizacional mediante el establecimiento de puentes (bridging) entre las diversas fuentes; esto es, en la transformación de los conocimientos en capacidad de conocer, 0 , dicho de otra forma, en la puesta en relación de la epistemología de la posesión con la de la práctica (Cook y Brown, 1999). Las dos fuentes fundamentales de información se condensan en otros tantos tipos de conocimiento, que, siguiendo la terminología de Karl Polanyi, podríamos describir como el explícito y el tácito. El primero está casi siempre organizado y es reconocible en soportes materiales o electrónicos, mientras que el segundo se presenta de forma desorganizada y difusa. El primero es el que predomina en la macropolítica, mientras que el segundo lo es en la micropolítica. A diferencia del carácter formal y sistemático del conocimiento explícito, el tácito es muy dependiente de la experiencia y del contexto, por lo que 
resulta difícil de formalizar y de comunicar ${ }^{5}$. Ambos son fuentes de información que se asientan en formatos distintos: la unidad del primero es la norma o el dato que fluye de arriba hacia abajo a lo largo de los cauces de autoridad y de los roles estructuralmente definidos; la del segundo es la experiencia, que fluye de forma horizontal a través de las redes de interacción.

Así como el conocimiento explicito se manifiesta siempre como estructura, el tácito lo hace como flujo. Pero es preciso advertir que, más que de dos tipos de conocimiento distintos, se trata de dos dimensiones complementarias del conocimiento que han de combinarse en la estrategia de un proyecto a seguir. La dimensión explícita puede ilustrarse con la imagen del mapa o del código de circulación que nos informan del relieve, de las distancias o de las normas que hay que cumplir en un desplazamiento; la tácita se correspondería con nuestra habilidad para leer el mapa, para orientarnos, para conducir mejor o peor o para elegir uno u otro camino. Casi cae por su propio peso observar que un grupo de expertos conductores y excursionistas, como un grupo de buenos profesores, no logrará la mejor opción si no consigue compartir y coordinar los conocimientos que alberga en las dos dimensiones, lo que resultará a todas luces imprescindible si la estrategia a seguir tiene un nuevo objetivo y no puede acomodarse al bagaje de las rutinas acumuladas. Es viejo el dicho de que cuatro ojos ven más que dos, pero es preciso observar que ese aumento de la información visualizada sólo es traducible en conocimiento útil cuando puede ponerse en común lo que todos ellos ven. Sin una comunicación eficaz, las visiones albergadas por las redes que constituyen los ojos de la organización no pueden coordinarse, y, por consiguiente, la gestión de las informaciones que procesan no puede traducirse en colaboración.

Como ocurre con muchos de los fenómenos de la micropolítica (con frecuencia asociados al cuchicheo o la charla de café), los procesos a través de los que fluye el conocimiento tácito tienden a ser menospreciados por el carácter informal de las situaciones en las que se originan y se trasmiten. Por eso creemos que puede resultar ilustrativo hacer referencia a dos contribuciones que en la misma perspectiva

${ }^{5} \mathrm{~A}$ la descripción de Cook y Brown cabría añadir las rutinas como estructuras prácticas que proporcionan conocimiento organizado y pautas de acción, tanto a nivel individual como colectivo. Sin embargo, la seguridad ontológica que proporcionan las rutinas - tal como entiende por ejemplo Anthony Giddens- sólo son eficaces en contextos estables y no favorecen actitudes adaptativas al cambio. 
interdisciplinar abierta por Polanyi resaltan el interés de la habilidad en gestionar ambos tipos de conocimiento, $y$, en especial, la importancia de no relegar a un lugar secundario lo que Polanyi denomina la dimensión tácita 0 el conocimiento personal. Los dos enfoques que vamos a mencionar muestran porqué el conocimiento a disposición de los individuos es la materia prima clave en la gestión de una organización.

El primer enfoque es el de Friedrich Hayek, para quien la desconfianza ante las rígidas planificaciones de las grandes organizaciones no deriva tanto de los vicios de la ley de hierro de las burocracias, cuanto de una equivocada visión del conocimiento social. Para Hayek todo problema organizativo es, en el fondo, un problema de alocación de recursos, y todo recurso es, en última instancia, un recurso conocido. Por tanto, todo problema organizativo puede analizarse como un problema de gestión del conocimiento (Hayek, 1997/1945; Terrén, 1997). No existe ningún talento ni equipo directivo que sea capaz de garantizar que posee toda la información para asegurar que su decisión es la más eficiente. En lo que parece guardar una clara sintonía con las observaciones de Weick y Luhmann anteriormente señaladas, para Hayek se produce siempre la constatación de un tipo de conocimiento espontáneo y disperso que se escapa a la planificación directiva, unida a la de la incertidumbre como un rasgo fundamental del entorno. La lógica del conocimiento práctico y cotidiano, sometida a un aprendizaje constante y a la adaptación a las circunstancias concretas y a situaciones únicas, escapa a la lógica acumulativa y sistematizadora del planificador, del experto 0 del directivo. Esto condena al fracaso cualquier tipo de liderazgo que sólo atienda a ese patrón de gobierno de la organización, pues las decisiones más válidas no son las que sólo se basan en la autoridad de aquellos, sino las que lo hacen en el método que mejor sepa utilizar el conocimiento disperso ${ }^{6}$.

El segundo enfoque afín que traemos a colación se centra en la cuestión de cómo se crea el conocimiento en las organizaciones. Al igual que la perspectiva de la teoría del conocimiento social de Hayek, estudiosos japoneses como Nonaka y Konno (1998) combaten la idea del

\footnotetext{
${ }^{6}$ Este método era para Hayek el mercado, porque pensaba más en la eficacia de la economía que en la de las organizaciones estatales, y porque pensaba también que la competencia era el mejor criterio de evaluación de la eficiencia. La eficiencia de las organizaciones educativas, al menos de las estatales, no puede dilucidarse en términos estrictamente mercantiles, lo que no quiere decir que haya que considerarlas ajenas a cualquier tipo de medición de su rendimiento.
} 
conocimiento como un proceso lineal y acumulativo. Ambos recurren al concepto de «Ba» (procedente de la filosofía de Kitaro Nishida) para designar el espacio compartido y multidimensional (físico, virtual, moral) del que emerge el conocimiento a través de un proceso de interacciones en espiral que entremezclan siempre conocimiento explícito y conocimiento tácito. El proceso en espiral es la senda de un aprendizaje permanente, siempre inacabado (lo que guarda mucha relación con la teoría del conocimiento de la epistemología evolutiva, pero quizás es poco afín a la visión del aprendizaje que subyace a la cultura escolar tradicional, tozudamente persistente).

Así como la teoría del uso del conocimiento social de Hayek nos permite extraer enseñanzas sobre cierta forma de ejercicio del liderazgo, en la descripción de este proceso de aprendizaje en espiral (inseparable como es de la acción en el marco de las prácticas comunicativas de las redes) puede verse el esquema básico de la creación del conocimiento organizacional que subyace a las visiones afines al modelo de la cultura colaborativa, como, por ejemplo, la de la organización que aprende (Bolívar, 2000) o la de la escuela total y su figura del profesor aprendiz (Hargreaves, 1996, p. 39) ${ }^{7}$. Senge $(1990$, p. 3) ya señaló a este respecto cómo las organizaciones que destacarán en el futuro serán las que fomenten formas nuevas y abiertas de pensar, y las que permitan que la gente aprenda cómo aprender conjuntamente. Si aprender es ante todo modificar el comportamiento en función de la información comunicada, parece plausible ver una organización que aprende como un sistema ${ }^{8}$ de comunicación muy sensible y capaz de procesar con fluidez las informaciones que se gestan en su seno.

${ }^{7}$ Para Fullan y Hargreaves (1991) el profesor total sólo puede desarrollarse en escuelas totales (de igual forma que el profesor aprendiz sólo puede desarrollarse en organizaciones que aprenden). Una escuela total es aquella que cuenta con una auténtica comunidad docente en la que se valora, se desarrolla y se apoya el juicio y la experiencia del profesorado.

${ }^{8}$ La noción de sistema aquí utilizada es la abierta y dinámica utilizada por Weick, aunque guarda una estrecha afinidad con el tipo de ensamblaje que Fernández Enguita (2003, pp. 96-103), siguiendo el modelo de Wilden (después de todo un modelo también basado en la comunicación), distingue de otros como el agregado o la estructura. De hecho, aunque muy escorado hacia el entorno externo, también su caracterización de sistema hace referencia al mayor nivel de complejidad interna que encierra la dirección y la apertura de una comunidad. 
Pero, para adaptarse a la incertidumbre y lanzarse con iniciativa a un cambio que no sea sólo reactivo sino creativo, las organizaciones deben alcanzar una capacidad colaborativa que no se logra con el desarrollo de la de aprender, sino que precisa de lo que el propio Senge denomina «compromiso». Eso es lo que las convierte en ese tipo especial de sistema que es la organización-comunidad.

\section{DE LA RED A LA COMUNIDAD: EL EFECTO SINERGÉTICO DEL CAPITAL SOCIAL INTRAORGANIZATIVO}

Podemos reformular ahora el interés de la micropolítica como la atención al ámbito en el que se han de establecer los puentes que permitan la coordinación entre las informaciones, las visiones y las disposiciones derivadas de las redes en una estrategia sinergética, esto es, en una conjunción activa y concertada de sus interacciones. En tal conjunción radica la clave de ese tipo de red de redes o metarred en la que consiste una organización-comunidad. Lo que hay que mostrar ahora es que, sin esa estrategia, la organización no puede dotarse de un proyecto y ganar confianza y compromiso con él, esto es, no puede desarrollarse como comunidad.

Utlilizando la metáfora del bridging ya descrita, podemos decir que el puente que permite esa estrategia sinergética se produce a través del capital social intraorganizativo, que se revela así como el engranaje fundamental de la micropolítica de la colaboración ${ }^{9}$ y como la materia prima fundamental sobre la que debe residir el gobierno de una organización que pretende cumplir un proyecto. Visto de esta forma, la clave estribaría en transformar la red de redes que es la organización en una comunidad.

Según Cohen y Prusak, el capital social se desarrolla a través de redes y de comunidades. La riqueza de las redes radica en su espontaneidad y en su flexibilidad o fácil adaptación al contexto (la riqueza de los lazos débiles de Granovetter). Pero, a diferencia de lo que ocurre en una

\footnotetext{
${ }^{9}$ Así lo muestran autores como Don Cohen y Lawrence Prusak (Cohen y Prusak, 2001), vinculados al I BM Institute for Knowledge M anagement. . Las líneas de investigación recogidas en la página web de este Instituto (y especialmente la referida a Knowledge socialization son representativas de esta tendencia, pues junto a capital social aparecen comunidades de práctica, intermediación y roles de conocimiento, historias para comunicar conocimiento tácito, etc. (http://www.research.ibm.com/knowsoc/project).
} 
comunidad, cuyo vínculo es más intenso, las redes no precisan implicación con un objetivo común que las trascienda, y exigen un tipo de compromiso que podríamos calificar de tenue. Como hemos visto, buena parte de lo que describimos como conocimiento tácito se gesta mediante las redes de interacción, a través sobre todo de las comunicaciones informales que caracterizan su tipo dominante de interacción. Pero, siendo fundamental el aporte de esta estructura social en red de la organización, una organización-proyecto requiere, además, unas pautas de actuación coordinada más sólidas (no más rígidas), y un tipo de interacción basado en un compromiso más firme con la organización y con sus objetivos. Requiere, en definitiva, un tipo de confianza distinto al que alimentan las redes. De ahí que puedan equipararse las dos formas de desarrollo del capital social con los dos tipos de confianza descritos por Robert Putnam : celgach y gnuesa(thin/thick). Conviene no olvidar que, como observó otro de los teóricos del capital social (Coleman, 1990, p. 310), el juego de las reciprocidades del que emerge esa confianza se desarrolla en paralelo con el intercambio de información, que con mucha frecuencia tiene lugar como resultado indirecto o como subproducto de interacciones comunicativas establecidas con otros fines.

Llegados a este punto, podemos establecer una equivalencia entre el logro de una organización eficiente en su gestión del conocimiento y con aptitud de dotarse de un adecuado potencial de conocer y de aprender, y el de una interacción fluida entre el conocimiento tácito y el explícito y el logro de una organización capaz de aprovechar sus redes internas para hacerlas converger en el proyecto que caracteriza a una organización-comunidad.

Por organización-comunidad entendemos aquella dotada no sólo de unos objetivos, sino también de un proyecto. La diferencia entre lo uno y lo otro consiste en que el cumplimiento de objetivos requiere destrezas, y sólo en segundo término actitudes o motivación, mientras que el de un proyecto necesita compromiso (resultado de una actitud de implicación). Mientras que lo primero puede lograrse con una motivación instrumental, lo segundo precisa ese tipo de motivación que describimos como comunitaria. Las destrezas (resultado de la información acumulada) son siempre necesarias, pero en momentos de cambio pueden resultar anacrónicas por rutinarias o insuficientes para reducir las nuevas entradas de incertidumbre. Como se muestra en los procesos de asesoría espontánea y de comunicación terapéutica, es entonces cuando las actitudes que emanan de la confianza y de los compromisos 
propios de las interacciones de una organización-comunidad muestran su potencial de generar nuevo conocimiento.

Las escuelas con un sólido capital social son sistemas de comunicación más sensibles y propensos a desarrollarse como comunidades. Interesa ver ahora en qué medida interviene la identidad de la organización en este proceso. Ayuda a ello la investigación sobre las comunidades de práctica. Wenger (1998), por ejemplo, considera que puede hablarse de una comunidad cuando existe un compromiso con una determinada práctica y cuando ese compromiso confiere identidad, es decir, cuando define la pertenencia a la organización. La tarea individual adquiere en este caso una dimensión colectiva a través de los procesos en que se gesta y se negocia. En atención a tales procesos, Wenger subraya la importancia de los procesos informales que caracterizan las experiencias de aprendizaje en la configuración de una organización como comunidad.

Ahora bien, en muchas ocasiones, sobre todo si el tamaño es grande o si las tareas están muy diversificadas porque existen muchos niveles o ciclos, los centros no parecen una comunidad de práctica, sino más bien una constelación de comunidades de práctica. En organizaciones como las escolares, los riesgos de que esta constelación se traduzca más en un escenario balcanizado que en una red de redes han sido descritos en la literatura sobre la cultura colaborativa. La balcanización puede considerarse como un obstáculo en la configuración de una organización-comunidad, porque impide que las comunidades de práctica se comuniquen en una red interactiva. Su efecto es, por tanto, el bloqueo de la puesta en marcha de procesos de comunicación organizacional que favorezcan esa motivación comunitaria en la que se basa la micropolítica de la colaboración que subyace a todo proyecto. La balcanización puede describirse como una estructura patológica de interacciones que alimenta posiciones contrarias o resistentes al cambio hacia la mejora, y que dificulta la adaptación del centro a las nuevas complejidades suministradas por los cambios del entorno. La balcanización de los claustros descrita por Hargreaves (1996), por ejemplo, genera una conflictividad latente y a menudo callada que bloquea su manifestación constructiva, pues, como se sabe (Ball, 1989, p. 36), la mayor parte de la comunicación cotidiana en el seno de las escuelas no refleja disputas de intereses sino interacciones no contradictorias.

Sin embargo, no es la balcanización de los claustros la única patología comunicativa que impide la gestión dinámica del conocimiento 
y el compromiso necesario para el cambio. Existen otros obstáculos más arraigados en el individualismo que en la fragmentación en redes. Según ha mostrado Hargreaves (1996), para que los proyectos de mejora se hagan con realismo y para que el cambio se ajuste a las verdaderas inquietudes y condiciones del contexto, es importante que prestemos atención tanto a los deseos de cambio del profesorado como a los de conservación y de estabilidad. Así, frente a las creencias y a las actitudes que propugnan la necesidad de colaborar para cambiar, hay que tener presentes otras, más infravaloradas en la investigación académica pero no menos habituales entre el profesorado, y de las que emergen interacciones resistentes al cambio impulsadas por una organizaciónproyecto o por una organización-comunidad. Estas actitudes son muy frecuentes en una institución tan conservadora como es la escuela (Rudduck, 1994), una organización robusta (Eisner,1992) en la que los roles tienden a ser considerados casi como eternos, en la que es normal que viejas prácticas persistan bajo cambios formales, y en la que la sujeción a rutinas establecidas proporciona seguridad y economía de esfuerzos. De ahí las actitudes que llevan a considerar las transformaciones hacia la mejora como reiterativas o como de escasa relevancia para el trabajo de aula; las que se miran como un mero ritual derivado de modas intelectuales o de operaciones políticas percibidas como poco conocedoras del mundo cotidiano de la escuela; y las que las rechazan porque las observan como si se tratara de un incremento escasamente reconocido (o retribuido) de las exigencias profesionales. Todo ello si se habla, además, de la posición (clásica en la literatura sobre la acción colectiva) del free-rider, que encuentra más racional beneficiarse de que los demás comparten y colaboran en el reajuste sin realizar él mismo el esfuerzo de compartir. Atender a las lógicas de acción basadas en estas actitudes y en las de su manifestación en las diversas redes de interacción intraorganizativas es importante para no imponer un proyecto sino negociarlo, y para saber acompasar yacomodar su ritmo de implementación a las diferentes velocidades de adaptación que exigen las disposiciones propias de cada red. ¿Cómo hacerlo?

\section{LAIMPORTANCIA DE LA COMUNICACIÓN COMO PROCESO MICROPOLÍTICO}

No vamos a ofrecer la respuesta definitiva. Pero sí intentaremos mostrar cómo a la vista de esos factores que bloquean la producción del capital social de la escuela, la consideración de ésta como un espacio de 
comunicación, es relevante a la hora de buscar y de analizar los caminos micropoliticos que pueden reforzar su capacidad colaborativa. En otras palabras, hemos de ver cómo la gestión de los procesos de comunicación en el seno de la organización es una variable fundamental a la hora de favorecer las redes internas de capital social, que permiten una gestión dinámica del conocimiento organizacional.

A estas alturas debe estar claro que la perspectiva de análisis de los centros educativos, que los presenta como espacios de comunicación, atiende ante todo a esa dimensión de la vida de la organización que es su cultura. Ya Robert Park señaló en los años veinte que la cultura es todo aquello que es comunicable. Proponemos también partir de la idea de que las organizaciones, como las sociedades, se reproducen a través de ese proceso comunicativo central en su vida cultural que es la opinión pública. En ella se entretejen y se negocian las expectativas y las actitudes de los miembros respecto a la organización como la imagen de ésta. La creciente valoración de tal dimensión de la vida organizativa se refleja con claridad en el hecho de que cada vez más consultorías y cursos de dirección ofrecen un lugar estelar al conocimiento de los procesos comunicativos, tanto en el seno de la propia organización como entre ella y su entorno. Suele prestarse mucha atención al problema de la selección más apropiada de los medios (una de cuyas versiones restringidas es la

denom inadaspeach communication of forma de dirigirse al público), que cada vez es más tenido en cuenta en la formación para el liderazgo. También es normal la preocupación por ese capítulo de las relaciones públicas que es la difusión de la imagen corporativa en relación con la identidad de la organización. Dichos procesos adquieren especial protagonismo en los momentos de turbulencia o de cambio, en los que una desorientación generalizada dificulta el liderazgo efectivo y erosiona la imagen de la organización, todo lo cual tiene que ver con lo que hoy ocurre con la enseñanza pública. En momentos como éstos la comunicación crítica aspira sobre todo a potenciar el diálogo y a reestablecer el orgullo profesional o el sentimiento de pertenencia, relajando las situaciones límite y renovando la imagen de la organización. Ambas son estrategias decisivas en la conformación de una organización-proyecto.

Parece que la comunicación es, en efecto, un elemento crucial a la hora de entender la realimentación informativa que nutre la capacidad cooperativa. El aislamiento que tantas veces se ha señalado en la investigación empírica sobre la práctica docente desde Lortie, se traduce en una serie de situaciones de incomunicación intraorganizativa, que, pervirtiendo la idea de la autonomía profesional, refuerza los muros 
de la privacidad y termina por institucionalizar el conservadurismo (Fullan y Hargreaves, 1992). Lo que resulta de ello es un déficit de información que dificulta la adaptación al cambio y una erosión de la confianza necesaria para lubricar las interacciones conflictivas pero constructivas, y fortalecer un sentimiento de pertenencia que cree identidad con el proyecto.

Aunque los trabajos pioneros de Herbert Simon en los años 50 ya habían llamado la atención sobre la relevancia de los sistemas de comunicación en las organizaciones (véase por ejemplo March y Simon, 1987, pp. 178-185), el estudio de la comunicación en el seno de las organizaciones ha ido cobrando importancia a medida que progresaba el interés por la vida cultural de la organización, y por desarrollar lo que se ha dado en llamar un modelo caliente o postburocrático de su funcionamiento (Hassard y Parker, 1993; Terrén, 2003).

La idea clave puesta a punto en los estudios de este nuevo campo de investigación (Kreps, 1990) es fundamental para el estudio de la micropolítica de la colaboración, pues señala que la comunicación es la herramienta fundamental en el ejercicio del liderazgo, en la consecución de un clima de cooperación en torno a un objetivo, en la elaboración y difusión de una imagen de la organización, y en el trasvase fluido de la información tanto de dentro hacia afuera como en el propio interior de la organización. Todas estas funciones son las que conforman el potencial de adaptación y la disposición al cambio de la organización, tan sensible a su funcionamiento como espacio de comunicación.

CUADRO 2

Formas de comunicación organizacional

\begin{tabular}{|c|c|c|c|}
\hline & Interna & Externa & \\
\hline \multirow{2}{*}{ Interpersonal } & Reuniones Comisiones & $\begin{array}{c}\text { Tutorías con alumnos o } \\
\text { con familias. Reuniones } \\
\text { con la administración o } \\
\text { con delegados } \\
\text { sindicales }\end{array}$ & Formal \\
\hline & $\begin{array}{c}\text { Conversaciones pasillo, } \\
\text { sala de profesores, } \\
\text { patio }\end{array}$ & $\begin{array}{l}\text { Conversaciones con } \\
\text { miembros de otros } \\
\text { centros, con la } \\
\text { administración o con } \\
\text { padres }\end{array}$ & Informal \\
\hline Mediada & Circulares Informes & $\begin{array}{l}\text { Publicidad (web, } \\
\text { folletos) }\end{array}$ & \\
\hline
\end{tabular}


La variada funcionalidad de la comunicación organizacional conduce a muy diversas formas de transmisión de mensajes, a formas de relevancia diferentes según los propósitos buscados. Esta diversidad aparece reflejada en el cuadro 2. La superposición de todas ellas es lo que contribuye a integrar a los individuos en la organización y a ésta en el entorno global en el que discurre su acción.

El hecho de que en nuestra sociedad de la información coexistan en la vida de un grupo o de una organización una gran diversidad de estrategias y de medios comunicativos, es lo que hace muy importante para la gestión de una organización la teoría de la selección de medios (Lucas, 1997, pp. 126 y ss.). Uno de los principios de esta teoría es que las formas comunicativas que hay que utilizar tienden a ser más esperadas, simples y rutinarias cuanto menos sensible, complejo o necesitado de efecto persuasivo es el mensaje. Así, parece apropiado comunicar mediante una circular las jornadas festivas del próximo curso, pero no un cambio de horarios, las observaciones formuladas por un inspector, la implementación de diversificaciones curriculares, o la programación de actividades que exigen una notable alteración de las rutinas cotidianas.

La investigación empírica sobre el liderazgo educativo muestra cómo, con mucha frecuencia, lo importante en su ejercicio no es formular objetivos cuanto elegir las estrategias comunicativas adecuadas a los diferentes individuos, contextos y momentos. Ahora puede verse mejor lo conveniente que resulta entender el ejercicio del liderazgo educativo desde la perspectiva del juego de influencias y no desde el ejercicio de la autoridad, y entender los entornos en los que se juega como complejos de informaciones. A ello pretende ayudar la clasificación reflejada en el cuadro 2. Así, dependiendo de si la comunicación se realiza con el entorno externo o entre miembros o segmentos de la propia organización, podemos distinguir entre las formas interna o externa de comunicación. Tanto en uno como en otro caso la comunicación puede ser interpersonal o mediada, según se efectúe en relaciones cara a cara o a través de al gún canal de intermediación. Las comunicaciones interpersonales, a su vez, pueden ser de tipo formal o informal.

Dada la estructura piramidal de la mayoría de las organizaciones burocráticas, la comunicación interna es muy sensible al estatus organizacional o a la posición que ocupan los emisores y receptores, pues eso es lo que condiciona que el flujo de la transmisión sea vertical (cuando discurre entre individuos cuyas posiciones se refieren a roles con diferente valoración jerárquica) u horizontal (sin diferencias de poder 
entre los polos comunicantes). La primera, normalmente formal, sigue los canales previstos en el organigrama y en los estatutos o reglamentos de la organización; la segunda, de carácter casi siempre informal, transita por interacciones no regladas, que pueden ir desde una charla de café entre profesores hasta el boca a boca de los padres en el momento de recoger al alumnado. El conocimiento que tiende a fluir a través de cada una de ellas es distinto.

Aunque la interacción comunicativa no es nunca una secuencia unidireccional, es cierto que en las organizaciones dotadas de una fuerte estructura burocrática suelen primar procesos lineales de tipo técnicamente autoritario, en los que los emisores presentan una posición de poder jerárquico frente a unos receptores concebidos como meros polos pasivos, que, a su vez, tienden a disimular su crítica, su escepticismo o su falta de gratificación percibida en los refugios informales de la resistencia no participativa que alimentan algunas redes. Sin embargo, cuando el estilo de la organización quiere cambiar hacia los model os más consultivos, participativos o democráticos que potencian la mejora del centro porque son capaces de gestionar (seleccionar y compartir) un m ayorym ejarstock de conocimientos, resultan más eficaces los procesos comunicativos que se conducen en espiral.

La comunicación interna de tipo formal suele consistir en la transmisión de instrucciones que tienen como objetivo el cumplimiento de las tareas propias de la organización. Es la más adecuada para la transmisión de ese tipo de conocimiento que describimos como conocimiento explícito. En este caso, el flujo de la información circula en sentido descendente, aunque en ocasiones pueda también hacerlo de forma ascendente (como cuando un tutor eleva un informe sobre su grupo o sobre un alumno al orientador 0 al coordinador). En cualquier caso, el sentido de la comunicación es vertical. La comunicación interna de tipo informal, por su parte, es la más relevante para el análisis de las redes de interacción intraorganizativa y para la traslación del conocimiento tácito. Está más afectada por lo que podríamos llamar la estructura sentimental de la organización: interacciones basadas en la empatía (la atracción, la curiosidad, la afinidad profesional o extraprofesional). Es un tipo de comunicación fundamental a la hora de calibrar el impacto de fenómenos como los rumores (flujos de información de origen no identificado), que tienen especial importancia en momentos de incertidumbre al interferir en la gestión de lo que describimos como comunicación crítica. Por eso, aunque casi siempre se produce en relaciones horizontales, puede superponerse a las verticales 
y alimentar o frenar el efecto de éstas, lo que afecta al ejercicio de funciones de guía y de coordinación del liderazgo.

Es la existencia de una fluida red de comunicaciones en el seno de la organización lo que permite una más eficaz gestión del conocimiento tácito y explícito, pues permite manejar y compartir una mayor cantidad de informaciones heterogéneas (unas organizadas en el conocimiento experto y representadas en bases de datos o legislativas, y, muchas de ellas, las relacionadas con el conocimiento tácito, generadas de forma ciega o espontánea). No es sólo tener mayor información sino compartir más lo que ayuda a el egir mejor. De ahí la importancia del logro deestaespeciede intranet, que permite la integración y la selección más dinámica de las informaciones y de las experiencias más adecuadas. Con ello se gana también una evaluación en tiempo real del curso del proyecto, y de la cantidad y del tipo de apoyos que suscita. Así, debe verse cómo la teoría de la comunicación intraorganizativa complementa la estrategia de producción del conocimiento en espiral descrita por la epistemología evolutiva, y por el modelo de producción del conocimiento en espiral de Nonaka y Konno.

Pero si, como señalamos, el compromiso que exige el proyecto del que se dota una organización-comunidad requiere un cierto sentimiento específico de identificación y de pertenencia, es preciso que reparemos en la importancia de la dimensión simbólica en la que se condensa esa identidad (la imagen de la organización), y en los procesos comunicativos que la alimentan en su interior. La proyección de la imagen corporativa que se realiza en la comunicación externa es sintetizada por Lucas Marín en la fórmula: imagen =identidad + comunicación. Interesa aquí porque el compromiso con la organización y la implicación en su funcionamiento están muy relacionados con el orgullo de la pertenencia a ella, y porque dicho orgullo e identificación están muy vinculados a la autoimagen que la organización sepa transmitir hacia adentro y negociar con sus redes. Pero, teniendo en cuenta que las reflexiones aquí expuestas han estado centradas en el interior de la organización, y que ello no significa (sino todo lo contrario) que las organizaciones entendidas como sistemas abiertos no se expanden como redes también hacia afuera, es importante reconocer hasta qué punto tal imagen percibida y reproducida en el interior es dependiente de la imagen que la organización sepa proyectar hacia el exterior. Esto es muy interesante para las instituciones que trabajan en un medio más o menos cuestionado por la opinión general, como es el caso de la educación pública. 
Aunque la proyección exterior de los centros educativos (al menos de los estatales) no es del todo igual a la de la mera imagen publicitaria y a la mercadotecnia de las organizaciones empresariales, sí puede entenderse en los términos de las relaciones públicas o de la comunicación de la imagen corporativa (más atenta a la identidad, al reconocimiento del prestigio y a la adecuación del servicio prestado que a la venta de un producto), y, desde luego, tiene claras repercusiones sobre la propia gestión de la imagen en el interior de la organización. Uno de los efectos de la patología comunicativa propiciada por la balcanización de los claustros, por ejemplo, es el deterioro de la imagen del centro, que se promueve como consecuencia de una reafirmación negativa de la identidad de los pequeños círculos. Esta reafirmación bloquea la circularidad de los procesos de trasvase de información, impide la renovación del sentimiento de pertenencia que parece exigir la motivación comunitaria de todo proyecto, y corta la espiral de mejora del centro.

La relación entre las proyecciones interna y externa de la imagen del centro es uno de los fenómenos que obliga a no perder de vista la conexión de los procesos comunicativos intraorganizativos con los extraorganizativos. Y es que la apertura de la organización hacia el interior no hace por sí sola que el sistema de la organización deje de poder ser cerrado hacia el exterior, sino que parece una condición necesaria para que deje de serlo. Climas organizativos abiertos que entretejen una fluida comunicación entre redes intraorganizativas no bastan para convertir un centro en una organización-proyecto, pues su misión no está orientada hacia sí misma sino hacia el público que conforma su entorno externo. Por muy fluida que sea la comunicación interna de una organización, ésta no podrá operar como un sistema abierto si ese tipo de comunicación no está alimentado por la comunicación externa entablada con otras organizaciones, con la administración o con el público. Sólo así puede armonizarse el aprendizaje desde dentro y desde afuera en un equilibrio productivo entre estabilidad e innovación o adaptación, en un contexto de cambio social acelerado y de constante interdependencia (Lucas, 1997, pp. 203-213). Por eso, la coordinación y la integración de los procesos de comunicación internos y externos (Kreps, 1990, pp. 24 y ss. ), la orquestación de colaboración y participación, son requisitos de las organizaciones escolares totales, tal como hemos visto que las describe Hargreaves. Esa integración puede estudiarse a partir de la hipótesis de que las organizaciones educativas con mayor capacidad de interacción comunicativa interna son también las que mayor capacidad de interacción comunicativa tienden a establecer con el entorno externo. 
0 , lo que es lo mismo, es poco probable que las escuelas puedan desarrollarse con eficiencia como redes en su interacción con el exterior si no lo hacen también en su interior a través de una micropolítica de la colaboración. Tal es la hipótesis sobre la que seguiremos trabajando.

\section{EN CONCLUSIÓN}

La exploración del enfoque micropolítico del estudio de las organizaciones aquí presentado, muestra el potencial analítico que resulta de la consideración de los centros educativos como sistemas de comunicación. La idea central defendida es que el análisis de los diferentes tipos de interacciones comunicativas que se registran en el interior de dichos sistemas, permite poner de manifiesto las diferentes formas en las que se produce la micropolitica de la organización escolar. Se ha mostrado cómo, a pesar del marco burocrático en el que se desarrolla la actividad escolar, la fundamentación teórica de una imagen caliente de la organización justifica que deba estudiarse como un orden negociado y en constante proceso, a través de las redes de interacción comunicativas que se describen en su seno. Aunque el enfoque propuesto se enmarca en la perspectiva del análisis cultural de las organizaciones, el trabajo ha mostrado también cómo, a la hora de estudiar la forma en que las organizaciones educativas se adaptan al cambio, eso no significa la utilización como variable dependiente de un concepto estático e invariable de cultura como conjunto de valores compartidos y previamente dados, tal como es frecuente hacer en los estudios del clima delas organizaciones. Antes bien, la atención a las prácticas comunicativas que fluyen en el universo de las redes de interacción intraorganizativas obliga a trabajar con una conceptual ización de la cultura en proceso que no puede derivarse de ninguna estructura dada, sino que debe verse en el marco de los intercambios (frecuentemente informales) del conocimiento tácito. Es en la micropolítica de dichos intercambios donde se fragua ese capital social interno, que permite responder a las incertidumbres generadas no sólo por los cambios del entorno externo a la organización, sino también a las producidas por el propio incremento de la complejidad y de la riqueza de las relaciones internas. Es en esa dialéctica donde se juega la fluidez comunicativa de la organización y su potencialidad para responder con iniciativa y compromiso (es decir, con un proyecto) a las nuevas incertidumbres; donde se juega, en definitiva, su flexibilidad adaptativa y su capacidad de aprendizaje. Por eso su análisis resulta de especial interés para el estudio del liderazgo y del 
cambio educativo, y, muy en especial, para el estudio de los procesos que subyacen a la conformación de culturas colaborativas en los centros educativos.

\section{BIBLIOGRAFÍA}

BACHARACH, S. B. y MUNDELL, M. (1981): «Organizational Politics in Schools: Micro, Macro and Logics of Action», en Educational Administration Quarterly, 29, 4, pp. 423-452.

BALL, S. (1989): La micropolítica de la escuela. Hacia una teoría de la organización escolar, Barcelona, Paidós.

BLASE, J. (ed.) (1991): The Politics of life in Schools: Power, Conflict and Cooperation, Newbury Park (Calif.), Sage. Madrid, La Muralla.

BoLívar, A. (2000): Los centros educativos como organizaciones que aprenden,

COHEN, D. y PRUSAK, L. (2001): «In Good Company: How Social Capital Makes Organizations Work», en Harvard Business Review Press, Boston.

Press.

Coleman, J . (1990): Foundations of Social Theory, Cambridge, Harvard University

CoOK, S. D. N. yBrown, J. S. (1999): «B ridging Epistemologies: the Generative Dance B etween Organizational Knowledge and Organizational Knowing», en Organization Science, 10, 4, pp. 381-400.

EISNER, E. W. (1992): «E ducational Reform and the Ecology of Schooling», en Teachers College Record, 93(4), pp. 610-621.

FERNÁNDEZ ENGUITA, M. (2003): «Organização escolar e modelo profissional», en Carapeto, N.S. (org. ), Formação continuada e gestão da educação, São Paulo, Cortez.

FULLAN, M. (1994): «La gestión basada en el centro: el olvido delo fundamental», en Revista de Educación, 304, pp. 147-161.

GIDDENS, A. (1984): La constitución de la sociedad, Buenos Aires, Amorrortu.

HARGREAVES, A. (1996): Profesorado, cultura y postmodernidad, Madrid, Morata. Londres, Sage.

HASSARD, J. y PARKER, M. (eds.) (1993): Postmodernism and Organizations,

Hayek, (1997/1945): «El uso del conocimiento en la sociedad», en REIS, 80, pp. 215-227. 
JARES, X. R. (1997): «El lugar del conflicto en la organización escolar», en Revista Iberoamericana de Educación, 15 (OEI, biblioteca digital).

KREPS, G. L. (1990): Organizational Communication, Nueva Cork, Longman. Madrid, Bosch

LUCAS MARÍN, A. (1997): La comunicación en la empresa y en las organizaciones, Nacional.

LUhmanN, N. (1983): Fe y racionalidad en los sistemas, Madrid, Editora

MARCH, J. G. y SIMON, H. (1987): Teoría de la organización, Barcelona, Ariel.

NONAKA, I. yKonno, N. (1998): «The Concept of ba: Building of Foundation for Knowledge Creation». California Management Review, 40, 3, pp. 40-54.

Akal.

RODRíguez Romero, M. (2003): Las metamorfosis del cambio educativo, Madrid,

RUDDUCK, J . (1994): «R eflexiones sobreel problema del cambio en las escuelas», en Angulo, F. y Blanco, N. (eds.): Teoría y desarrollo del currículum, Málaga, Archidona.

Santos Guerra, M. A. (1997): La luz del prisma para comprender las organizaciones, Málaga, Aljibe.

TERRÉN, E. (1997): «La inevitable imperfección del conocimiento humano: Hayek y el uso social del conocimiento», en REIS, 80, pp. 205-214.

- (2003): «Rutina, diversidad eincertidumbre: la organización educativa ante entornos multiculturales», en N. Carapeto (ed.): A gestão da educação na sociedade mundializada, D P\&A editora, Rio de Janeiro, pp. 127-154.

Wenger, E. (1998): Communities of Practice: Learning, Meaning and Identity, Cambridge University Press, Cambridge, MA. 